\title{
INTRODUCTION \\ Operative imaging and visualization: cutting edge techniques and future directions
}

\author{
Paul Larson, MD, ${ }^{1}$ Peter Nakaji, MD, ${ }^{2}$ Walter Stummer, MD, PhD, ${ }^{3}$ and John Pollina, MD ${ }^{4}$ \\ 1Department of Neurological Surgery, University of California, San Francisco, California; '2Department of Neurosurgery, \\ Banner-University Medical Center, Phoenix, Arizona; ${ }^{3}$ Department of Neurosurgery, Münster University Hospital, Münster, \\ Germany; and ${ }^{4}$ Department of Neurosurgery, State University of New York at Buffalo, New York
}

I $\mathrm{T}$ is hard to image anything that has had more of an impact on our specialty over the years than advancements in imaging and visualization of the brain and spine. From early use of the operative microscope, to the advent of CT and MRI, and most recently the development of contemporary neuronaviation systems, the ability to "see" relevant anatomy and pathology has allowed neurosurgeons to perform procedures that would have seemed technically impossible just a few decades ago. These advancements in visualization have expanded our therapeutic options and vastly improved clinical outcomes for our patients.

This issue of Neurosurgical Focus explores the next generation of operative imaging and visualization techniques. New surgical planning tools show promise for improving patient safety and surgical efficiency. Visualization techniques such as intraoperative fluorescence and advanced neuroendoscopy can provide real-time feedback during demanding intracranial and spinal procedures. Finally, the advent of augmented reality gives surgeons the potential for an environment in which imaging and direct visualization can coexist in an immersive, unobtrusive way.

Novel techniques and technologies from 11 countries spanning Europe, Asia, Africa, and the Americas are represented in this issue. Some are mature, others are still at the proof-of-principle stage, but all of them give us an exciting glimpse into our future.

https://thejns.org/doi/abs/10.3171/2020.10.FOCUS20910

\section{Disclosures}

Dr. Larson reports being an unpaid, noncontract consultant for ClearPoint Neuro, Inc. Dr. Nakaji reports direct stock ownership in GT Medical Technologies; being a patent holder for OsteoMed; receiving royalties from OsteoMed; and being a consultant for True Digital Surgery. Dr. Stummer reports being a consultant for NXDC and Photonamic, and being on the speakers bureau for Medac. Dr. Pollina reports being a consultant for Medtronic and ATEC.

\section{Correspondence}

Paul Larson: paul.larson@ucsf.edu. 